\title{
A Method for Tractable Dynamical Studies of Single and Double Shock Compression
}

E.J. Reed, L.E. Fried, M.R. Manaa, J.D. Joannopoulos

This article was submitted to

$13^{\text {th }}$ American Physical Society Topical Conference on Shock Compression of Condensed Matter

Portland, OR, July 20-25, 2003

\section{July 18, 2003}

U.S. Department of Energy

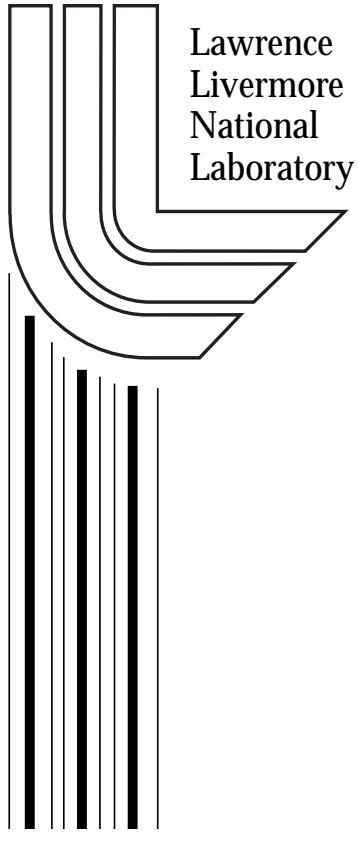




\section{DISCLAIMER}

This document was prepared as an account of work sponsored by an agency of the United States Government. Neither the United States Government nor the University of California nor any of their employees, makes any warranty, express or implied, or assumes any legal liability or responsibility for the accuracy, completeness, or usefulness of any information, apparatus, product, or process disclosed, or represents that its use would not infringe privately owned rights. Reference herein to any specific commercial product, process, or service by trade name, trademark, manufacturer, or otherwise, does not necessarily constitute or imply its endorsement, recommendation, or favoring by the United States Government or the University of California. The views and opinions of authors expressed herein do not necessarily state or reflect those of the United States Government or the University of California, and shall not be used for advertising or product endorsement purposes.

This is a preprint of a paper intended for publication in a journal or proceedings. Since changes may be made before publication, this preprint is made available with the understanding that it will not be cited or reproduced without the permission of the author.

This report has been reproduced directly from the best available copy.

Available electronically at http://www.doc.gov/bridge

Available for a processing fee to U.S. Department of Energy

And its contractors in paper from

U.S. Department of Energy

Office of Scientific and Technical Information

P.O. Box 62

Oak Ridge, TN 37831-0062

Telephone: (865) 576-8401

Facsimile: (865) 576-5728

E-mail: reports@adonis.osti.gov

Available for the sale to the public from

U.S. Department of Commerce

National Technical Information Service

5285 Port Royal Road

Springfield, VA 22161

Telephone: (800) 553-6847

Facsimile: (703) 605-6900

E-mail: orders@ntis.fedworld.gov

Online ordering: http://www.ntis.gov/ordering.htm

OR

Lawrence Livermore National Laboratory

Technical Information Department's Digital Library

http://www.llnl.gov/tid/Library.html 


\title{
A method for tractable dynamical studies of single and double shock compression
}

\author{
Evan J. Reed*, Laurence E. Fried ${ }^{\dagger}$, M. Riad Manaa ${ }^{\dagger}$ and J. D. Joannopoulos* \\ *Department of Physics, Massachusetts Institute of Technology, Cambridge, MA 02139 \\ ${ }^{\dagger}$ Chemistry and Materials Science Directorate, Lawrence Livermore National Laboratory, Livermore, CA \\ 94550
}

\begin{abstract}
A new multi-scale simulation method is formulated for the study of shocked materials. The method combines molecular dynamics and the Euler equations for compressible xow. Treatment of the diffcult problem of the spontaneous formation of multiple shock waves due to material instabilities is enabled with this approach. The method allows the molecular dynamics simulation of the system under dynamical shock conditions for orders of magnitude longer time periods than is possible using the popular non-equilibrium molecular dynamics (NEMD) approach. An example calculation is given for a model potential for silicon in which a computational speedup of $10^{5}$ is demonstrated. Results of these simulations are consistent with the recent experimental observation of an anomalously large elastic precursor on the nanosecond timescale.
\end{abstract}

\section{INTRODUCTION}

Molecular dyanmics simulations have provided valuable insight into atomic scale dynamical processes in shock waves.[1, 2, 3, 4] However, existing methods of performing these simulations are generally limited to the 10 picosecond timescale. The popular nonequilibrium molecular dynamics (NEMD) approach to atomistic simulations of shock compression involves creating a shock on one edge of a large system and allowing it to propagate until it reaches the other side. The computational work required by NEMD scales at least quadratically in the evolution time because larger systems are needed for longer simulations. When quantum mechanical methods with poor scaling of computational effort with system size are employed, this approach to shock simulations rapidly becomes impossible. Another approach that utilizes a computational cell moving at the shock speed has the same drawbacks.[5] This paper presents a method which circumvents these diffculties by requiring simulation only of a small part of the entire system. The effects of the shock wave passing through this small piece of the system are simulated by dynamically regulating the applied stress which is obtained from a continuum theory description of the shock wave structure. Because the size of the molecular dynamics system is independent of the simulation time in this approach, the computational work required to simulate a shocked system is nearly linear in the simulation time. By circumventing the scaling problems of NEMD, molecular dyanmics simulation of shocked materials for orders of magnitude longer timescale becomes possible.

Molecular dynamics simulations have been performed that utilize a shock Hugoniot-based thermodynamic constraint for the temperature at £xed volume.[6] This approach is a thermodynamic one for a single shock wave and fails to capture the spontaneous formation of multiple shock waves and dynamical effects like long-lived metastable phases, elastic-plastic phase transitions and chemical reactions, which are ubiquitous in shocked condensed matter. The new method outlined in this paper is a method for the dynamical simulation of shock waves that solves these problems. It enables the dynamical simulation of shock waves in systems that have material instabilities which lead to the formation of multiple shock waves and chemical reactions that can change the speed of shock propagation with time. It is a tractable method that requires no a priori knowledge of the system phase diagram, metastable states, 
or sound speeds.

\section{SIMULATION OF A SINGLE SHOCK WAVE}

We model the propagation of the shock wave using the 1D Euler equations for compressible dow, which neglect thermal transport. These equations represent the conservation of mass, momentum, and energy respectively everywhere in the wave. Neglecting thermal transport in high temperature shocks is valid in systems where electronic mechanisms of heat conduction are not important, i.e. usually less than a few thousand $\mathrm{K}$ in insulators.[7] While continuum theory is not rigorously applicable at elastic shock fronts, the correct dynamics will be approximated in these special regions. We seek solutions of these equations which are steady in the frame of the shock wave moving at speed $\mathrm{v}_{\mathrm{s}}$. This substitution, and integration over $x$ yields a variation of the Hugoniot relations,

$$
\begin{array}{r}
u=\mathrm{v}_{\mathrm{s}}\left(1-\frac{\rho_{0}}{\rho}\right), \\
p-p_{0}=\mathrm{v}_{\mathrm{s}}{ }^{2} \rho_{0}\left(1-\frac{\rho_{0}}{\rho}\right), \\
e-e_{0}=p_{0}\left(\frac{1}{\rho_{0}}-\frac{1}{\rho}\right)+\frac{\mathrm{v}_{\mathrm{s}}^{2}}{2}\left(1-\frac{\rho_{0}}{\rho}\right)^{2} .
\end{array}
$$

Here $u$ is the local speed of the material in the laboratory frame (particle velocity), $v$ is the specifc volume, $\rho=1 / v$ is the density, $e$ is the energy per unit mass, and $p$ is the negative component of the stress tensor in the direction of shock propagation, $-\sigma_{x x}$. Variables with subscripts 0 are the values before the shock wave, and we have chosen $u_{0}=0$, i.e. the material is initially at rest in the laboratory frame. In the language of shock physics, Eq. 2 for the pressure is the Rayleigh line and Eq. 3 for the internal energy is the Hugoniot at constant shock velocity. These equations apply to a system which has a timeindependent steady-state in the reference frame moving at the shock speed $\mathrm{v}_{\mathrm{s}}$.

For the molecular dynamics simulation, we employ the Lagrangian,

$L=T\left(\left\{\xi_{i}\right\}\right)-V\left(\left\{\vec{r}_{i}\right\}\right)+\frac{1}{2} Q \Psi^{2}+\frac{1}{2} \frac{\mathrm{v}_{\mathrm{s}}^{2}}{v_{0}^{2}}\left(v_{0}-v\right)^{2}+p_{0}\left(v_{0}-v\right)$ where $T$ and $V$ are kinetic and potential energies per unit mass, and $Q$ is a mass-like parameter for the simulation cell size. It can be seen that Eq. 4 implies Eq. 3 when $\phi=0$ because $T+V=e$. The equation of motion for the system volume is,

$$
Q \ddot{v}=\frac{\partial T}{\partial v}-\frac{\partial V}{\partial v}-p_{0}-\frac{\mathrm{v}_{\mathrm{s}}^{2}}{v_{0}^{2}}\left(v_{0}-v\right)
$$

which reduces to Eq. 2 when $\ddot{v}=0$. We use the scaled atomic coordinate scheme of Ref [8] to deal with the variable computational cell size. This scheme introduces a volume dependence for $T$ and $V$. Strain is only allowed in the shock direction, i.e. $v_{0}-v=$ $-\varepsilon_{x x} v_{0}$ where $\varepsilon_{x x}$ is the uniaxial strain. The pressures in Eq. 5, including the thermal contribution, are taken to be the uniaxial $x$ component of stresses. Computational cell dimensions transverse to the shock direction are £xed, as in NEMD simulations. This approach allows the simulation of shocks propagating in any direction which is diffcult or impossible with NEMD.

Simulation of a single shock wave may be accomplished by dynamically varying the uniaxial strain of the system according to Eq. 5. By choosing a small representative sample of the shocked material, it is assumed that stress gradients and thermal gradients in the actual shock wave are negligible on the length scale of the sample size. While the thermal energy is assumed to be evenly distributed throughout the sample, thermal equilibrium is not required. It can be shown that the stable states of the constraint equations satisfy shock wave stability requirements. [10]

To simulate a shock to a given pressure, the initial state parameters which de£ne the MD constraint in Eq. 4 are chosen $\left(\rho_{0}, p_{0}, e_{0}\right.$.) A guess for $\mathrm{v}_{\mathrm{s}}$ is made for the constraint to take the system to the desired £nal pressure. If the £nal pressure is other than the desired one, improved guesses for $\mathrm{v}_{\mathrm{s}}$ can be made and simulated again until the desired $\mathrm{v}_{\mathrm{S}}$ is determined. The £nal shock pressure increases with increasing $\mathrm{v}_{\mathrm{s}}$. The simulation of a shock to a given particle velocity using this approach is a straightforward extension. 


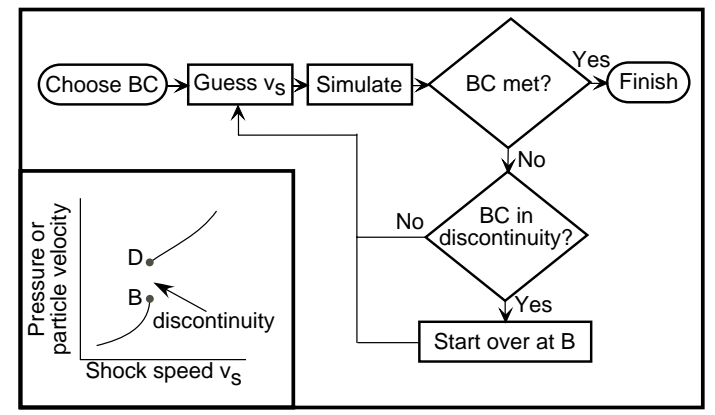

FIGURE 1. Flowchart for simulation of a shock to a chosen pressure or particle velocity boundary condition (BC). Instabilities due to regions where $\frac{d^{2} p}{d v^{2}}<0$ along the Hugoniot can give rise to a discontinuity in the inset plot.

\section{TREATMENT OF MULTIPLE SHOCK WAVES}

The above method describes the simulation of a single stable shock wave. However, it is not always possible to shock to a given pressure or particle velocity using this technique. For example, it may not be possible to connect a straight Rayleigh line to all £nal pressures when there is a region of negative curvature in the Hugoniot, $\frac{d^{2} p}{d v^{2}}<0$. Such regions of negative curvature are common in condensed phase materials and may be a result of phase transformations or may be the shape of a single phase Hugoniot. While a single Rayleigh line is insuffcient to meet the pressure boundary condition in this region, two Rayleigh lines are suffcient.

Figure 1 shows a rowchart that illustrates how to determine the set of Rayleigh lines that are stable and meet the boundary conditions without any a priori knowledge of the system. A shock wave instability exists when the boundary condition falls within a discontinuity in the set of £nal pressures as a function of shock speed, as in the inset fgure in Figure 1. The existence of such a discontinuity can be determined when suffcient trial values of $\mathrm{v}_{\mathrm{S}}$ have been simulated. If the boundary condition falls within the discontinuity, the entire process is repeated with point $\mathrm{B}$ as the initial state to fnd the shock speed that meets the boundary condition. If further instabilities are discovered that prevent the boundary condition from being met with a single shock, the process is continued.
The formation and evolution of multiple waves becomes more complicated when chemical reactions or phase transitions occur. Volume decreasing phase transformations cause the pressure at point B in Figure 1 to decrease with time. Parameterization of the $p$-v space path with Rayleigh lines is valid when the timescale of this pressure change is less than the time required for a material element to reach the Enal shocked state. This condition can be made rigorous though the so-called shock change equation and shown to hold for times longer than some timescale. [10]

\section{APPLICATION TO SILICON}

As an illustrative example, we apply the new method to an elastic-plastic transition in a model potential for silicon. Figure 2 shows shock speed as a function of particle velocity for shock waves propagating in the [011] direction in silicon described by the Stillinger-Weber potential.[11] This potential has been found to provide a qualitative representation of condensed properties of silicon. Data calculated using the NEMD method are compared with results of the new method presented in this paper. NEMD simulations were done with a computational cell of size $920 \AA \times 12 \AA \times 11 \AA$ unit cells (5760 atoms) for a duration of about 10-20ps. Simulations with the new method were done with a computational cell size of $19 \AA \times 12 \AA \times 11 \AA$ unit cells (120 atoms). Both simulations were started at $300 \mathrm{~K}$ and zero stress. Since the NEMD simulations were limited to the 10ps timescale by computational cost, simulations with the new method were performed to calculate the Hugoniot on this 10ps timescale for comparison. The £nal particle velocity in these simulations was taken to be a point of steady state after a few ps.

Figure 2 indicates a single shock wave exists below $1.9 \mathrm{~km} / \mathrm{sec}$ particle velocity. Above this particle velocity, an elastic shock wave preceeds a slower moving shock characterized by plastic deformation. Agreement between the two methods is good for all regions except for the plastic wave speed for particle velocities less than $2.1 \mathrm{~km} / \mathrm{sec}$. The wide range of values for the plastic wave speeds in NEMD simulations in this regime is due to fnite simulation cell size effects. Better agreement in this regime can be obtained by using simulation cells with larger cross 


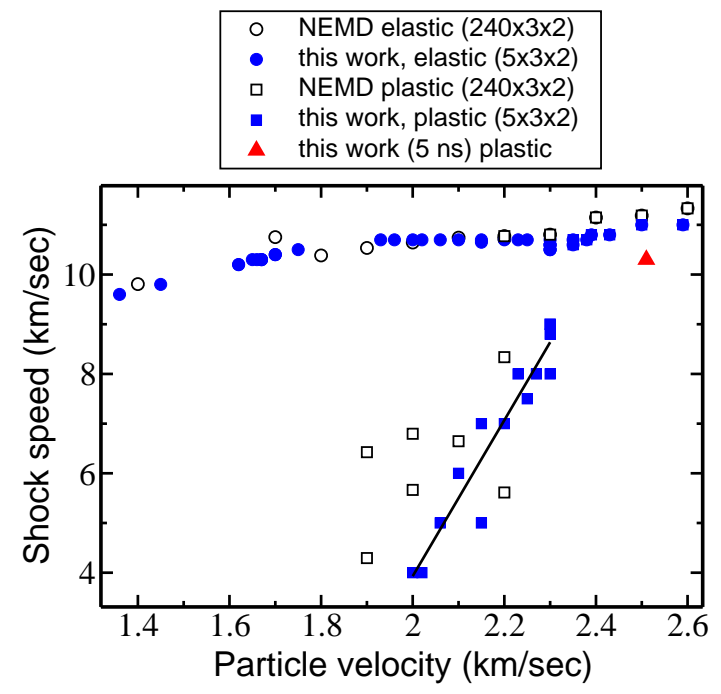

FIGURE 2. Comparison of calculated Hugoniots for the NEMD approach and the method presented in this paper for roughly $10 \mathrm{ps}$ runs. Note the ability to utilize much smaller computational cell sizes with the new method. Also included is one data point for a $5 \mathrm{~ns}$ simulation using this work which would be prohibitive with NEMD requiring a factor of $10^{5}$ increase in computational effort.

sectional area.

One of the primary advantages of using the method outlined in this paper is the ability to simulate for much longer times than is possible with NEMD. As an example, Figure 2 shows the result of a 5 ns simulation performed along a Rayleigh line corresponding to a shock speed of $10.3 \mathrm{~km} / \mathrm{sec}$. The uniaxially compressed elastic state required $5 \mathrm{~ns}$ to undergo plastic deformation. The difference in particle velocity between the $10 \mathrm{ps}$ and $5 \mathrm{~ns}$ simulations at this shock speed is $0.8 \mathrm{~km} / \mathrm{sec}$, suggesting that the elastically compressed state is metastable with an anomalously large lifetime. This is consistent with experimental observations of shocked silicon that indicate an anomalously high pressure elastic wave exists on the nanosecond timescale.[12] In addition to the simulations performed with the Stillinger-Weber potential, we have performed more accurate tightbinding[13] 120 atom simulations using the method of this paper that also suggest an anomalously high pressure elastic wave precursor exists on the $10 \mathrm{ps}$ timescale.

This simulation done with NEMD would require more than $5 \mathrm{~ns}$ simulation time due to the time required for the equilibration of the Erst and second wave speeds. For an $\mathscr{O}(\mathscr{N})$ method of force evaluation, the computational cost of this simulation with the NEMD method would be at least $10^{5}$ times greater, and therefore not tractable.

\section{ACKNOWLEDGMENTS}

We thank J. Forbes, C. Tarver, and D. Hare for helpful discussions. Evan Reed acknowledges support from the Department of Defense NDSEG Fellowship and the Lawrence Livermore National Laboratory MRI/EMC graduate fellowship. This work was performed under the auspices of the US Department of Energy by the University of California, LLNL under contract number W-7405-Eng-48.

\section{REFERENCES}

1. Kadau, K., Germann, T. C., Lomdahl, P. S., and Holian, B. L., Science, 296, 1681 (2002).

2. Germann, T. C., Holian, B. L., Lomdahl, P. S., and Ravelo, R., Phys. Rev. Lett., 84, 5351 (2000).

3. Kress, J. D., Bickham, S. R., Collins, L. A., Holian, B. L., and Goedecker, S., Phys. Rev. Lett., 83, 3896 (1999).

4. Holian, B. L., and Lohmdahl, P. S., Science, 280, 2085 (1998).

5. Zhakhovski1, V. V., Zybin, S. V., Nishihara, K., and Anisimov, S. I., Phys. Rev. Lett., 83, 1175 (1999).

6. Maillet, J. B., Mareschal, M., Soulard, L., Ravelo, R., Lomdahl, P. S., Germann, T. C., and Holian, B. L., Phys. Rev. E, 63, 016121 (2001).

7. Zel'dovich, Y. B., and Raizer, Y. P., Physics of shock waves and high-temperature hydrodynamic phenomena, Academic Press, New York, NY, 1967.

8. Allen, M. P., and Tildesley, D. J., Computer simulation of liquids, Oxford University Press, New York, 1989.

9. Duvall, G. E., "," in Proceedings of the International School of Physics, Physics of High Energy Density, Academic Press, New York, 1971, p. 7.

10. Reed, E. J., Fried, L. E., and Joannopoulos, J. D., Phys. Rev. Lett., 90, 235503 (2003).

11. Stillinger, F. H., and Weber, T. A., Phys. Rev. B, p. 5262 (1985).

12. Loveridge-Smith, A., Allen, A., Belak, J., Boehly, T., Hauer, A., Holian, B., Kalantar, D., Kyrala, G., Lee, R. W., Lohmdahl, P., Meyers, M. A., Paisley, D., Pollaine, S., Remington, B., Swift, D. C., Weber, S., , and Wark, J. S., Phys. Rev. Lett., 86, 2349 (2001).

13. Sawada, S., Vacuum, 41, 612 (1990). 